\title{
Asthma control and medication adherence among asthmatic outpatients in Vietnam: A cross-sectional study
}

\author{
Sang Thanh Huynh ${ }^{1,2}$, Ngoc Viet Nguyen ${ }^{2,3}$, Ho Nhu Nguyen²* \\ 1 Department of Clinical Pharmacy, School of Pharmacy, Ho Chi Minh City University of Technology (HUTECH University), Ho Chi Minh City, Vietnam \\ 2 Department of Clinical Pharmacy, School of Pharmacy, University of Medicine and Pharmacy at Ho Chi Minh City, Ho Chi Minh City, Vietnam \\ 3 Department of Global Public Health, Karolinska Institutet, Stockholm, Sweden
}

\begin{abstract}
Suboptimal asthma control and medication non-adherence are common and associated with adverse disease outcomes. This study aimed to assess the medication adherence, level of asthma control, and determine factors associated with asthma control among asthmatic outpatients. A cross-sectional study was conducted on 250 asthmatic patients aged $\geq 12$ visiting the Asthma Management Clinic at a tertiary hospital in Vietnam from April to June 2019. Data, including demographics, medication treatment and adherence, and asthma control, were collected through structured interviews and prescription review. Asthma control and medication adherence were measured using the Asthma Control Test (ACT) questionnaire and the Morisky Medication Adherence Scale 8items (MMAS-8), respectively. Multivariable logistic regression analysis was used to determine the associated factors with asthma control. The mean age of the study participants was 59.1 years and the majority were women $(67.2 \%)$. All patients were using inhaled corticosteroid (ICS)-containing controller therapy. The proportion of patients highly adhering to prescribed medications was $34.4 \%$. Well-controlled asthma was observed in $64.4 \%$ of patients. Age group of $\geq 60(\mathrm{aOR}=3.43,95 \% \mathrm{CI} 1.21-9.68)$, low medication adherence $(\mathrm{aOR}=2.97,95 \% \mathrm{CI}$ 1.40-6.30), and using medium or high dose of ICS (aOR=3.38, 95\% CI 1.64-6.97; aOR=4.28, 95\%CI 1.90-9.65, respectively) were associated with poorly-controlled asthma. Poorly controlled asthma was still prevalent. Patient's age, medication adherence, and ICS dosage are some factors which healthcare providers should consider when asthma control was under expectation.
\end{abstract}

Keywords:

Asthma, Asthma control, Asthma control test, Medication adherence, Inhaled corticosteroid, Vietnam

\section{INTRODUCTION}

Asthma is a heterogeneous disease of which the main characteristic is chronic airway inflammation which results in respiratory symptoms such as cough, wheezing, shortness of breath, and chest tightness ${ }^{1}$. Asthma is a major global public health concern with approximately 339 million people affected in 2018 and 1,000 people dying due to asthma-related problems every $\mathrm{day}^{2}$. In Vietnam, asthma remains a health and economic burden. The estimated prevalence among adults ranged from $2.4 \%-6.0 \%$ in 2011 and the total cost of asthma was 23,165 billion Vietnam Dong (VND) in 2014 ${ }^{3-6}$.

According to the Global Initiative for Asthma
(GINA), the important goals of asthma management are to gain good control of asthma symptoms and to minimize the risk of future asthma-related consequences ${ }^{1}$. Uncontrolled asthma is associated with many adverse outcomes, namely decreased lung function, increased risk of life-threatening asthma exacerbation, poor quality of life, and increased treatment cost ${ }^{1}$. To achieve asthma control, pharmacological controller therapy is of critical importance. Inhaled corticosteroids (ICS) are strongly recommended by the GINA guidelines as a crucial component in the controller therapy for all adolescents and adults with asthma ${ }^{1,7}$. Despite the advances in management, the availability of established treatment guidelines, and effective treatment therapy, poorly-controlled asthma

*Corresponding author:

*Ho Nhu Nguyen Email: nhnguyen@ump.edu.vn 
is a common problem globally and in Vietnam. The CHAS study conducted on 2,159 adult patients with asthma in Spain found a prevalence of uncontrolled asthma of $63.9 \%{ }^{8}$. In an Italian study on 995 patients with asthma, $32.7 \%$ of participants had poor disease control. Clinicians' low adherence to GINA treatment guidelines was highly prevalent $(71.2 \%)$ and strongly associated with poor asthma control ${ }^{9}$. In Vietnam, a 2017 cross-sectional study reported only $40.4 \%$ of asthmatic patients having the disease under good control ${ }^{10}$.

The GINA guidelines recommend periodical asthma control assessment in the disease management cycle $^{1,7}$. Numerical tools for assessing the level of asthma control, including the Asthma Control Test (ACT), are commonly used in clinical research ${ }^{1,7}$. Asthma control is a multifaceted issue and could be affected by many factors as shown in previous studies. These factors might include age, sex, socio-economic status, smoking, environmental factors, comorbidity, medication treatment, and adherence ${ }^{11}$. In asthmatic patients, poor medication adherence has been reported in many studies with the prevalence of optimal adherence of only $30-50 \%{ }^{7,12,13}$. Medication non-adherence has been shown to be associated with adverse disease outcomes, including poor disease control, increased risk of exacerbations, increased healthcare costs, decreased lung function, and asthma deaths ${ }^{7,12,13}$. Studying potentially associated factors with asthma control, including medication adherence, may be of great help when it comes to developing interventions to improve the treatment outcomes ${ }^{14-16}$.

The greatly growing pace of population and economics in Ho Chi Minh City (HCM City), which is one of the most important regions for healthcare in Vietnam, is posing a threat to asthmatic health. Currently, there is limited information about the level of asthma control and factors that may affect this level. Therefore, we aimed to investigate the medication use, medication adherence, asthma control, and determine associated factors with asthma control among patients with asthma at a tertiary hospital in HCM City.

\section{MATERIALS AND METHODS}

\subsection{Study design and population}

This was a descriptive cross-sectional study. Study participants were outpatients with an asthma diagnosis aged 12 years old and older visiting the Asthma Management Clinic at a 1,500-bed tertiary hospital in HCM City from March 01, 2019 to June 30, 2019.

For sample size calculation, the following formula was used:

$$
\mathrm{n} \geq \frac{z_{(1-\alpha / 2)}^{2} \times p(1-p)}{d^{2}}
$$

where $\mathrm{d}$ is the desired margin of error $(5 \%), \mathrm{z}$ is 1.96 corresponding to a $95 \%$ confidence interval, and $p$ is prior judgement of the correct value of the proportion of poorly controlled asthma in the study population. We chose $p=0.156$ based on a study conducted in six Asian countries, including Vietnam, by Chiu KC. et al. ${ }^{17}$. The study used the Asthma Control Test to assess asthma control and reported the proportion of poorly controlled asthma of $15.6 \%$.

Patients who had chronic obstructive pulmonary disease, were pregnant or breastfeeding, or refused to participate were excluded from the study. We invited potentially eligible patients during the study period to participate in the study. Written informed consents were obtained prior to starting the data collection process. We received the ethical approval from the Ethics Committee of the study hospital before the start of the study (Approval reference number 51/CN-HDDD. Date of approval: June 21, 2019).

\subsection{Data collection}

We designed and pre-tested a structured questionnaire before the study commencement. The following information was included in the questionnaire: demographic characteristics (age, sex, education level), disease-related information (comorbidities, family history, body mass index-BMI, smoking history), pharmacological treatment (controller therapy, medication adherence), and level of asthma control. ICS dose level (low/medium/high) was ascertained using the 2019 GINA guideline ${ }^{7}$. Patients' medication adherence was measured using the Morisky Medication Adherence Scale-eight items (MMAS-8). The Asthma Control Test (ACT) questionnaire was used to assess the level of asthma control. Both instruments had been translated and validated for studies conducted in Vietnam and in Vietnamese patients ${ }^{18}$.

The ACT questionnaire assesses the level of asthma control during the previous four weeks. It includes five items asking about symptoms, use of rescue medication, daily functioning, and self-assessment. The score for each question ranges from 1 to 5 and the total score is between 5 and 25 . Higher scores indicate better asthma control. A total ACT scores of "20-25", "16-19", and "5-15" correspond to "well-controlled asthma", "not well-controlled asthma", and "very poorly-controlled asthma", respectively ${ }^{19}$. In terms of medication adherence assessment, an MMAS-8 score of 8 indicates "high adherence", 6 to $<8$ indicates "medium adherence", and $<6$ indicates "low adherence" 20.

Face-to-face interviews with eligible participants during their hospital visits for asthma were conducted by two well-trained interviewers using the structured questionnaire to collect the study data. The interviews were protocol-guided. Patients' most recent prescriptions were also examined to collect information on medication therapy that patients were using and to 
double-check some information obtained through patient interviews, including age, BMI, and comorbidity. The prescriptions were provided by patients during the interviews.

\subsection{Statistical analysis}

We performed a descriptive analysis of the collected data. Frequencies and percentages were reported for categorical variables. Means and standard deviations or median and interquartile range were reported for continuous variables. Multivariable logistic regression analysis was used to determine the associated factors with the level of asthma control. The dependent variable was the asthma control level assessed by the ACT questionnaire. Participants were divided into two groups, namely well-controlled (ACT score $\geq 20$ ) and poorly-controlled (ACT score <20). The latter was the outcome of interest in this analysis. The independent variables included age group, sex, education level, BMI,

Table 1. Patients' demographics and disease-related information $(\mathrm{N}=250)$.

\begin{tabular}{|c|c|c|c|}
\hline Patient characteristics & & Frequency (n) & Percentage (\%) \\
\hline \multirow[t]{4}{*}{ Age } & Mean age: $59.1 \pm 13.7$ & & \\
\hline & $12-44$ & 35 & 14.0 \\
\hline & $45-59$ & 76 & 30.4 \\
\hline & $\geq 60$ & 139 & 55.6 \\
\hline \multirow[t]{2}{*}{ Sex } & Male & 82 & 32.8 \\
\hline & Female & 168 & 67.2 \\
\hline \multirow[t]{4}{*}{ Education } & Primary school & 31 & 12.4 \\
\hline & Secondary school & 62 & 24.8 \\
\hline & High school & 56 & 22.4 \\
\hline & College/university & 101 & 40.4 \\
\hline \multirow[t]{2}{*}{ Family history of asthma } & No & 153 & 61.2 \\
\hline & Yes & 97 & 38.8 \\
\hline \multirow[t]{3}{*}{ Number of comorbidities } & 0 & 41 & 16.4 \\
\hline & $1-3$ & 146 & 58.4 \\
\hline & $>3$ & 63 & 25.2 \\
\hline \multirow[t]{4}{*}{ Comorbidity } & Allergic rhinitis & 76 & 30.4 \\
\hline & Gastro-esophageal reflux disease (GERD) & 33 & 13.2 \\
\hline & Cardiovascular disease & 49 & 19.6 \\
\hline & Arthritis & 42 & 16.8 \\
\hline \multirow[t]{3}{*}{ BMI $\left(\mathrm{kg} / \mathrm{m}^{2}\right)$} & $<23$ & 101 & 40.4 \\
\hline & $23-<25$ & 72 & 28.8 \\
\hline & $\geq 25$ & 77 & 30.8 \\
\hline \multirow[t]{2}{*}{ Current smoking $^{\dagger}$} & Yes & 85 & 34.0 \\
\hline & No & 165 & 66.0 \\
\hline
\end{tabular}

†Including active smoking and secondhand smoke exposure.

Table 2. Asthma pharmacological treatment $(\mathrm{N}=250)$.

\begin{tabular}{|c|c|c|}
\hline Pharmacological treatment & Frequency (n) & Percentage $(\%)$ \\
\hline \multicolumn{3}{|l|}{ ICS dose } \\
\hline Low dose & 83 & 33.2 \\
\hline Budesonide (mean daily dose, $\mu \mathrm{g} \pm \mathrm{SD}$ ) & $269 \pm 75$ & \\
\hline Fluticasone (mean daily dose, $\mu \mathrm{g} \pm \mathrm{SD}$ ) & $188 \pm 65$ & \\
\hline Medium dose & 110 & 44.0 \\
\hline Budesonide (mean daily dose, $\mu \mathrm{g} \pm \mathrm{SD}$ ) & $633 \pm 33$ & \\
\hline Fluticasone (mean daily dose, $\mu \mathrm{g} \pm \mathrm{SD}$ ) & $500 \pm 0$ & \\
\hline High dose & 57 & 22.8 \\
\hline Budesonide (mean daily dose, $\mu \mathrm{g} \pm \mathrm{SD}$ ) & N/A & \\
\hline Fluticasone (mean daily dose, $\mu \mathrm{g} \pm \mathrm{SD}$ ) & $1000 \pm 0$ & \\
\hline \multicolumn{3}{|l|}{ Controller therapy } \\
\hline ICS+LABA+LTRA & 118 & 47.2 \\
\hline $\mathrm{ICS}+\mathrm{LABA}$ & 100 & 40.0 \\
\hline ICS+LABA+LTRA+OCS & 15 & 6.0 \\
\hline ICS & 6 & 2.4 \\
\hline $\mathrm{ICS}+\mathrm{LABA}+\mathrm{OCS}$ & 6 & 2.4 \\
\hline Others* & 3 & 1.2 \\
\hline Missing & 2 & 0.8 \\
\hline
\end{tabular}

*Other therapies include: ICS+LTRA ( $n=1)$, ICS+LABA+LTRA+tiotropium (n=1), ICS+LABA+theophylline $(n=1)$.

ICS: inhaled corticosteroids; LABA: long-acting beta-2 agonists; LTRA: Leukotriene receptor antagonists; OCS: oral corticosteroids. 
smoking status, family history, number of comorbidities, allergic rhinitis, ICS dose, and medication adherence. All statistical analyses were performed using Statistical Package for Social Sciences software (Version 23.0. Armonk, NY: IBM Corp.) with the significance level of $5 \%$.

\section{RESULTS}

\subsection{Demographics and disease-related information}

There were 250 patients included in the study with a mean age of $59.1 \pm 13.7$. The majority of patients were female $(67.2 \%)$ and over 60 years old $(55.6 \%)$. Most patients had at least one comorbidity $(83.6 \%)$, of which allergic rhinitis was the most frequent one $(30.4 \%)$. Nearly $60 \%$ of patients were overweight or obese (BMI $\left.\geq 23 \mathrm{~kg} / \mathrm{m}^{2}\right)$. Current smoking was common with a prevalence of $34.0 \%$. Table 1 summarized patients' demographics and disease-related information.

\subsection{Asthma controller therapy}

ICS was indicated to all patients in the study (100.0\%). Regarding ICS dose level, medium-dose was most frequently used $(44.0 \%)$, followed by low-dose $(33.2 \%)$ and high dose $(22.8 \%)$. Budesonide and fluticasone were the only ICSs prescribed.

Long-acting beta-2 agonists (LABA) (97.2\%) and leukotriene receptor antagonists (LTRA) (54.4\%) were other most commonly prescribed medications. Oral corticosteroids were used by $8.5 \%$ of patients, and no patients were prescribed with biological drugs. Almost all patients (97.6\%) were using combination therapy, in which ICS+LABA+LTRA (47.2\%) and ICS+ LABA $(40.0 \%)$ were the two most common regimens, respectively. Details about pharmacological treatment were presented in Table 2.

\subsection{Medication adherence}

The mean score of MMAS- 8 was $6.4 \pm 1.7$. Participants' responses to each item in the MMAS-8 were presented in Table 3. The prevalence of high, medium, and low level of adherence was $34.4 \%, 38.4 \%$ and $27.2 \%$, respectively.

\subsection{Level of asthma control and associated factors}

The mean ACT score of study participants was 20.4 +3 .9. Asthma was well-controlled in $64.4 \%$ of the patients. Patients with very poorly-controlled disease accounted for $13.6 \%$. Results of the asthma control assessment using the ACT questionnaire were illustrated in Table 4.

We used multivariable logistic regression analysis to determine the association between the level of asthma control and demographic characteristics, disease-related and treatment-related information. Table 5 showed the results of the analysis. Age, ICS dose, and medication adherence were found to be statistically associated with asthma control. Patients aged 60 years or over (adjusted $\mathrm{OR}=3.43,95 \% \mathrm{CI} 1.21-9.68$ ), prescribed with medium or high dose of ICS (adjusted OR=3.38, 95\%CI 1.64-6.97; adjusted OR=4.28, 95\%CI 1.90-9.65, respectively), with low level of medication adherence (adjusted OR=2.97, 95\%CI 1.40-6.30) had higher odds of poorly-controlled asthma (all with $p$-value $<0.05$ ).

\section{DISCUSSION}

Our study investigated the characteristics of

Table 3. Participants' medication adherence assessed using MMAS-8 (N=250).

\begin{tabular}{|c|c|c|}
\hline MMAS-8 & $\begin{array}{l}\text { Yes response } \\
\text { n }(\%)\end{array}$ & $\begin{array}{l}\text { No response } \\
\mathbf{n}(\%)\end{array}$ \\
\hline Item 1: Do you sometimes forget to take your medications? & $105(42.0)$ & $145(58.0)$ \\
\hline $\begin{array}{l}\text { Item 2: People sometimes miss taking their medications for reasons other than forgetting. Thinking } \\
\text { over the past two weeks, were there any days when you did not take your medications? }\end{array}$ & $76(30.4)$ & $174(69.6)$ \\
\hline $\begin{array}{l}\text { Item 3: Have you ever cut back or stopped taking your medications without telling your doctor, } \\
\text { because you felt worse when you took it? }\end{array}$ & $25(10.0)$ & $225(90.0)$ \\
\hline Item 4: When you travel or leave home, do you sometimes forget to bring along your medications? & $46(18.4)$ & $204(81.6)$ \\
\hline Item 5: Did you take your medications yesterday? & $30(12.0)$ & $220(88.0)$ \\
\hline $\begin{array}{l}\text { Item 6: When you feel like your health condition is under control, do you sometimes stop taking } \\
\text { you medications? }\end{array}$ & $80(32.0)$ & $170(68.0)$ \\
\hline $\begin{array}{l}\text { Item 7: Taking medications every day is a real inconvenience for some people. Do you ever feel } \\
\text { hassled about sticking to your treatment plan? }\end{array}$ & $35(14.0)$ & $215(86.0)$ \\
\hline $\begin{array}{l}\text { Item 8: How often do you have difficulty remembering to take all your medications? (Once in a } \\
\text { while/ Sometimes/ Usually/ All the time=Yes, Never/rarely=No) }\end{array}$ & $29(11.6)$ & $221(88.4)$ \\
\hline Mean MMAS-8 score & $6.4 \pm 1.7$ & \\
\hline High level (MMAS-8=8) (n, \%) & $86(34.4)$ & \\
\hline Medium level (MMAS-8=6-7) $(\mathrm{n}, \%)$ & $96(38.4)$ & \\
\hline Low level (MMAS-8<6) (n, \%) & $68(27.2)$ & \\
\hline
\end{tabular}

MMAS-8: Morisky Medication Adherence Scale 8-items. 
Table 4. Level of asthma control assessed by ACT questionnaire $(\mathrm{N}=250)$.

\begin{tabular}{lccc}
\hline Level of asthma control & Frequency $(\mathbf{n})$ & Percentage $(\%)$ & ACT score $($ Mean \pm SD) \\
\hline Well-controlled & 161 & 64.4 & $22.8 \pm 1.5$ \\
Not well-controlled & 55 & 22.0 & $18.0 \pm 1.1$ \\
Very poorly-controlled & 34 & 13.6 & $12.7 \pm 2.2$ \\
\hline
\end{tabular}

Table 5. Multivariable logistic regression analysis on factors associated with poorly-controlled asthma.

\begin{tabular}{lccc}
\hline Factors & Adjusted OR & $\mathbf{9 5 \%}$ CI & p \\
\hline Sex (female vs. male) & 0.78 & $0.42-1.44$ & 0.425 \\
\hline Age group (vs. 12-44 years old) & & & 0.091 \\
45-59 years old & 2.48 & $0.87-7.09$ & 0.020 \\
$\geq 60$ years old & 3.43 & $1.21-9.68$ & 0.490 \\
\hline Education (high school or higher vs. below high school) & 0.81 & $0.44-1.48$ & 0.509 \\
\hline BMI $\left(\geq 25\right.$ vs. $<25 \mathrm{~kg} / \mathrm{m}^{2}$ ) & 0.80 & $0.42-1.54$ & 0.995 \\
\hline Smoking (yes vs. no) & 0.99 & $0.54-1.86$ & 0.081 \\
\hline Family history (yes vs. no) & 1.71 & $0.94-3.10$ & 0.671 \\
\hline Number of comorbidities (vs. 0) & & & 0.623 \\
$1-3$ & 0.79 & $0.34-1.90$ & \\
$>3$ & 0.81 & $0.30-2.17$ & 0.001 \\
\hline Allergic rhinitis (yes vs. no) & 0.85 & $0.44-1.63$ & \\
\hline ICS dose (vs. low dose) & & & \\
Medium dose & 3.38 & $1.64-6.97$ & \\
High dose & 4.28 & $1.90-9.65$ & \\
\hline Medication adherence (vs. high adherence) & & & \\
Medium & 1.50 & $0.75-3.00$ & 0.258 \\
Low & 2.97 & $1.40-6.30$ & 0.005 \\
\hline
\end{tabular}

Note. Poorly-controlled asthma is the combination of not well-controlled and very poorly-controlled asthma.

medication use, assessed the medication adherence and asthma control, and identified the factors associated with asthma control among asthma outpatients at a tertiary hospital in Vietnam. Regarding medication use, all patients were using ICS-containing controller therapy. Similarly, a very high prevalence of ICS use among patients with asthma was also reported in a study conducted in Vietnam by Diep TVD. et al. in $2016(96.4 \%)^{21}$. This is well in line with the 2019 GINA guideline which recommends the use of ICS in the controller therapy for all asthma patients aged 12 or older as soon as possible after diagnosis ${ }^{7}$. ICS is a mainstay of asthma treatment and has shown to reduce the risk of severe exacerbations and to improve symptom control ${ }^{7,22}$. Similar to results by Diep TVD. et al., the majority of patients in our study were using low- and medium-dose ICS ${ }^{21}$. According to GINA, low to medium doses of ICS are adequate for most patients with asthma to fully achieve clinical benefits ${ }^{7}$. Consistent with a previous study by Lin et al. ${ }^{23}$, ICS+LABA+LTRA and ICS+LABA were the two most common combinations for controller therapy indicated in our study. ICS+LABA is the preferred controller therapy recommended by the 2019 GINA guideline for patients at treatment step 3 or above. The use of the combination of ICS+LABA+LTRA has been becoming very prevalent among patients with asthma despite to the 2019 GINA guideline, this combination is only recommended for patients whose asthma is not controlled with a 3-month treatment with medium or high dose ICS+LABA at treatment step 4. More data on treat- ment steps and cooperation with doctors would be needed if we want to assess the rationale behind the use of controller therapy.

About medication adherence, the majority of patients did not adhere well to the prescribed medications. This result is similar to studies by Smits D. et al. and by Chiu KC. et al. which reported high proportions of non-adherence $(65.3 \% \text { and } 81.1 \% \text {, respectively })^{17,24}$. In addition, the MMAS-8 instrument was able to address the reasons behind non-adherence of patients. In our study, many intentional reasons were observed in the participants. For example, 32\% of the participants would stop taking medications when they assumed the condition was under control or felt worse with the treatment. The patients could also miss doses for reasons other than forgetting. This finding suggests enhancing patient education on medication use and adherence with a focus on the importance of long-term medication adherence and management of medication side effects. Medication adherence plays a crucial role in the treatment of diseases, especially chronic diseases such as asthma. Many studies have shown that non-adherence to asthma medications was associated with poor asthma control, increased risk of exacerbations, decreased lung function, impaired work productivity, increased healthcare costs, and high rates of asthma mortality ${ }^{14-16}$. In addition, if healthcare professionals fail to address patient's compliance issues, they may misjudge the patient's condition and this could lead to unnecessary ICS dose increases or drug additions. The consequences 
are an increased risk of side effects and increased treatment costs for patients.

The goal of asthma management is to achieve asthma control ${ }^{1}$. The prevalence of well-controlled asthma in our study was $64.4 \%$. In comparison with previous studies which used the same assessment tool, this figure is similar to the result of a study by Chiu KC. et al. (64.4\%) but higher than that of studies by Nguyen NV. et al. (40.4\%) and Diep TVD. et al. (59.1\%) $10,17,21$. Our study identified age, ICS dose, and medication adherence as factors associated with asthma control. In the adjusted logistic regression model, patients aged $\geq 60$ had 3.4 times higher odds of poor asthma control compared to those aged 12-44. This finding is consistent with results from previous studies although the thresholds used to define age groups among those studies and ours are slightly different. In the 2011 National Asthma Survey in the US ${ }^{25}$, elderly patients ( $\geq 65$ years old) had worse asthma control compared to patients aged 18-64 years old (OR=1.50; 95\% CI 1.12-2.01). A prior study by Nanshan Z. et al. showed that patients aged $\geq 45$ years old had a $23 \%$ higher odds of poor asthma control $(\mathrm{OR}=1.23$; $95 \%$ CI $1.07-1.41)^{26}$. Poor disease control is a common issue among elderly patients with asthma. There are several possible explanations for this phenomenon. Compared to younger patients, elderly patients with asthma have decreased lung function and usually respond less effectively to asthma medications, including ICS and bronchodilators ${ }^{7,27}$. Also, multimorbidity, including arthritis and cardiovascular diseases which were documented in our study samples, is a common problem in elderly individuals ${ }^{7}$. These diseases along with asthma can cause daily activity limitation which is a criterion in the ACT tool. In addition, elderly patients are more likely to have impaired cognition and wrong inhaler technique ${ }^{1,7}$. The aforementioned factors altogether can make asthma more difficult to treat in elderly patients.

Our study found that patients prescribed with medium- or high-dose ICS had higher odds of poorlycontrolled asthma compared to those receiving low-dose ICS. A study by Marjolein P. et al. also identified highdose of ICS as an independent predictor for poorer asthma control $(p=0.04)^{28}$. A longitudinal observational study over a 12-year period by Iida Vähätalo et al. documented that the use of higher ICS doses was significantly more common in patients with uncontrolled asthma throughout the follow-up period ${ }^{29}$. One possible explanation is that patients with uncontrolled disease might be prescribed with higher doses of ICS as a way to improve the disease control level. According to GINA guidelines ${ }^{7}$, higher doses of ICS may be required for more severe asthma but in most cases, low- to medium-dose ICS is adequate for a full clinical benefit. However, increasing ICS dose does not necessarily help improve disease control. In fact, as we checked the previous prescriptions of participants, most of those with highdose ICS had been prescribed with this dose without deescalation for at least 1-3 months prior to assessment date. Also, higher doses of ICS are commonly associated with an increased risk of adverse effects. Our result reemphasizes the importance of assessing the treatment issues that can affect asthma control, including doctors' adherence to GINA treatment guidelines, uncontrolled comorbidity, wrong inhaler technique, and medication non-adherence, before titrating up ICS dose for uncontrolled asthma ${ }^{1,7,9}$.

In our study, patients with a low level of medication adherence had three times higher odds of poor asthma control compared to highly adherent patients. This result is consistent with many previous studies. A cross-sectional study conducted on 1,054 asthma patients in six countries in Asia found that patients with high or medium adherence had a significantly higher prevalence of well-controlled asthma (67\% vs. $61 \%, p=0.042)^{17}$. In the study by Janez ic $\mathrm{A}$. et al..$^{30}$, an improvement in adherence from low to medium or from medium to high was associated with $65 \%$ higher odds of well-controlled asthma (adjusted $\mathrm{OR}=1.65, p=0.027$ ). These two studies used the same tools to measure asthma control and medication adherence as our study which are the ACT questionnaire and the MMAS-8, respectively. Medication non-adherence is an important risk factor for not only poor asthma control but also exacerbations ${ }^{7,12,13}$. Therefore, medication adherence assessment is recommended at every hospital visit of asthma patients. Since only $34.4 \%$ of patients in our study were highly adherent, health professionals should pay more attention to this treatment aspect in order to enhance adherence and improve treatment outcomes.

There are some limitations that should be acknowledged in our study. Firstly, the results might not be generalized to all asthma patients in Vietnam but may serve as preliminary insights to take further steps for improving medication adherence and treatment outcome. Secondly, this was a cross-sectional study, thereby only allowing us to determine the association between asthma control and the investigated factors. Longitudinal studies would be needed if we want to infer the causality. In addition, data on asthma control and medication adherence might be subject to misclassification bias as we used self-reported questionnaires. Also, patients might have given more socially desirable answers for medication adherence which could lead to overestimation of the adherence level. However, ACT and MMAS- 8 were valid and reliable tools that have been widely used in research.

\section{CONCLUSIONS}

In conclusion, the present study revealed that suboptimal medication adherence and inadequately- 
controlled asthma were still prevalent in Vietnam. There were associations between poor asthma control and older age, higher doses of ICS, and low level of medication adherence. Interventions to improve asthma control and patient care should be implemented with a focus on enhancing treatment adherence and tailoring to elderly patients.

\section{Conflict of interest \\ None.}

\section{Funding}

The study was supported by research grants from the University of Medicine and Pharmacy at Ho Chi Minh City, with grant number 24/2019/HD-DHYD. This funding source had no role in study design, data collection and analysis, decision to publish, or preparation of the manuscript.

\section{Ethics approval}

None to declare.

\section{Author contributions}

The author's responsibilities were as follows: STH, NVN, HNN: study concept and desgin; STH, NVN: acquisition of data; STH, NVN, HNN: analysis and interpretation of data; STH, NVN: statistical analysis; STH: drafting of the manuscript; STH, NVN, HNN: critical revision of manuscript. All authors read and approved the final manuscript.

\section{Article info:}

Received August 29, 2021

Received in revised form December 18, 2021

Accepted December 21, 2021

\section{REFERENCES}

1. Global Initiative for Asthma (GINA). Global Strategy for Asthma Management and Prevention [document on the Internet]. 2021 [cited 2021 Dec 12]. Available from: https://ginasthma.org/wpcontent/uploads/2021/05/GINA-Main-Report-2021-V2-WMS.pdf.

2. Global Initiative for Asthma (GINA). Global Strategy for Asthma Management and Prevention Online Appendix [document on the Internet]. GINA; 2018 [updated 2018; cited 2019 July 15]. Available from: https://ginasthma.org/wp-content/uploads/2018/03/ WMS-FINAL-GINA-2018-Appendix_v1.3.pdf.

3. Lam HT, Ronmark E, Tu'o'ng NV, Ekerljung L, Chuc NT, Lundback B. Increase in asthma and a high prevalence of bronchitis: results from a population study among adults in urban and rural Vietnam. Respir Med. 2011;105(2):177-85.

4. Tram TV. Asthma at Tien Giang province. Ho Chi Minh City Med J. 2011;15:

5. Duong SQ, Hoang BMT, Nguyen TQ, Hung NV, Quynh Nhu DT, Bao NQ, et al. Prevalence of asthma and asthma-like symptoms in Dalat Highlands, Vietnam. Singapore Med J. 2007;48(4):294-303.

6. Nguyen TTT, Nguyen NBT. Economic Burden of Asthma in Vietnam: An Analysis from Patients' Perspective. Value Health. 2014;17(7):A627.

7. Global Initiative for Asthma (GINA). Global Strategy for Asthma Management and Prevention [document on the Internet]. 2019 [cited 2019 July 6]. Available from: https://ginasthma.org/wpcontent/uploads/2019/04/GINA-2019-main-Pocket-Guide-wms. pdf.

8. González Barcala FJ, de la Fuente-Cid R, Álvarez-Gil R, Tafalla $\mathrm{M}$, Nuevo J, Caamaño-Isorna F. Factores asociados con el control del asma en pacientes de atención primaria en España: el estudio CHAS. Arch Bronconeumol. 2010;46(7):358-63.

9. Baldacci S, Simoni M, Maio S, Angino A, Martini F, Sarno G, et al. Prescriptive adherence to GINA guidelines and asthma control: An Italian cross sectional study in general practice. Respir Med. 2019;146:10-7.

10. Nguyen VN, Huynh TTH, Chavannes NH. Knowledge on selfmanagement and levels of asthma control among adult patients in Ho Chi Minh City, Vietnam. Int J Gen Med. 2018;11:81-9.

11. Lourido-Cebreiro T, Facal D, Rodriguez-Garcia C, GonzalezBarcala F-J. Asthma Control: A Continuing Challenge. Open Respir Arch. 2020;2:5-6.

12. Vlasnik JJ, Aliotta SL, DeLor B. Medication adherence: factors influencing compliance with prescribed medication plans. Case manager. 2005;16(2):47-51.

13. De Smet BD, Erickson SR, Kirking DM. Self-reported adherence in patients with asthma. Ann Pharmacother. 2006;40(3):414-20.

14. Williams LK, Pladevall M, Xi H, Peterson EL, Joseph C, Lafata $\mathrm{JE}$, et al. Relationship between adherence to inhaled corticosteroids and poor outcomes among adults with asthma. J Allergy Clin Immunol. 2004;114(6):1288-93.

15. Engelkes M, Janssens HM, de Jongste JC, Sturkenboom MC, Verhamme KM. Medication adherence and the risk of severe asthma exacerbations: a systematic review. European Respir J. 2015;45(2):396-407.

16. Levy ML. The national review of asthma deaths: what did we learn and what needs to change?. Breathe (Sheff). 2015;11:14-24.

17. Chiu KC, Boonsawat W, Cho SH, Cho YJ, Hsu JY, Liam CK, et al. Patients' beliefs and behaviors related to treatment adherence in patients with asthma requiring maintenance treatment in Asia. J Asthma. 2014;51(6):652-9.

18. Nguyen VN, Chavannes N, Le LTT, Price D. The Asthma Control Test (ACT) as an alternative tool to Global Initiative for Asthma (GINA) guideline criteria for assessing asthma control in Vietnamese outpatients. Prim Care Respir J. 2012;21:85-9.

19. Nathan RA, Sorkness CA, Kosinski M, Schatz M, Li JT, Marcus P, et al. Development of the asthma control test: a survey for assessing asthma control. J Allergy Clin Immunol. 2004;113(1): 59-65.

20. Morisky DE, Ang A, Krousel-Wood M, Ward HJ. Predictive validity of a medication adherence measure in an outpatient setting. J Clin Hypertens (Greenwich). 2008;10(5):348-54.

21. Diep TVD, Dang NDT. Investigation on medication use and factors associated with levels of asthma control among asthmatic outpatients at University Medical Center Hochiminh City. Pharm Sci Asia. 2018;45(1):13-21.

22. Busse WW, Pedersen S, Pauwels RA, Tan WC, Chen YZ, Lamm CJ, et al. The Inhaled Steroid Treatment As Regular Therapy in Early Asthma (START) study 5-year follow-up: effectiveness of early intervention with budesonide in mild persistent asthma. J Allergy Clin Immunol. 2008;121(5):1167-74.

23. Lin J, Fu X, Jiang P, Song W, Hu X, Jie Z, et al. Post hoc analysis of initial treatments and control status in the INITIAL study: an observational study of newly diagnosed patients with asthma. BMC Pulm Med. 2020;20(1):87.

24. Smits D, Brigis G, Pavare J, Maurina B, Barengo NC. Factors related to good asthma control using different medical adherence scales in Latvian asthma patients: an observational study. NPJ Prim Care Respir Med. 2017;27:39.

25. Talreja N, Baptist AP. Effect of age on asthma control: results from the National Asthma Survey. Ann Allergy Asthma Immunol. 2011;106(1):24-9.

26. Zhong N, Lin J, Zheng J, Lai K, Xie C, Tang K-J, et al. Uncontrolled asthma and its risk factors in adult Chinese asthma 
patients. Ther Adv Respir Dis. 2016;10(6):507-17.

27. Reed CE. Asthma in the elderly: diagnosis and management. J Allergy Clin Immunol. 2010;126(4):681-7.

28. De Vries MP, van Den Bemt L, Lince S, Muris JWM, Thoonen BPA, van Schayck CP. Factors Associated with Asthma Control. J Asthma. 2005;42(8):659-65.
29. Vähätalo I, Ilmarinen P, Tuomisto LE, Niemelä O, Kankaanranta H. Inhaled corticosteroids and asthma control in adult-onset asthma: 12-year follow-up study. Respir Med. 2018;137:70-6.

30. Janežič A, Locatelli I, Kos M. Criterion validity of 8-item Morisky Medication Adherence Scale in patients with asthma. PloS One. 2017;12(11):e0187835. 